\title{
Measurement of the spectroscopy of orbitally excited B mesons at LEP
}

\section{L3 Collaboration}

M. Acciarri ${ }^{\mathrm{z}}, \mathrm{P}$. Achard ${ }^{\mathrm{s}}$, O. Adriani ${ }^{\mathrm{p}}, \mathrm{M}$. Aguilar-Benitez ${ }^{\mathrm{y}}, \mathrm{J}$. Alcaraz ${ }^{\mathrm{y}}$, G. Alemanni v, J. Allaby ${ }^{\mathrm{q}}$, A. Aloisio ${ }^{\mathrm{ab}}$, M.G. Alviggi ${ }^{\mathrm{ab}}$, G. Ambrosi ${ }^{\mathrm{s}}$, H. Anderhub au , V.P. Andreev f,aj, T. Angelescu ', F. Anselmo i, A. Arefiev aa, T. Azemoon ${ }^{\text {c }}$, T. Aziz ${ }^{j}$, P. Bagnaia ai ${ }^{\text {, L. Baksay }}{ }^{\text {ap }}$, A. Balandras ${ }^{\text {d }}$, R.C. Ball ${ }^{\text {c }}$, S. Banerjee ${ }^{j}$, Sw. Banerjee ${ }^{j}$, A. Barczyk ${ }^{\text {au,as }}$, R. Barillère ${ }^{q}$, L. Barone ai P. Bartalini v, M. Basile ${ }^{\text {i }, ~ R . ~ B a t t i s t o n ~}{ }^{\text {af }}$, A. Bay ${ }^{\text {v }}$, F. Becattini ${ }^{\text {p }}$, U. Becker ${ }^{\text {n, }}$ F. Behner ${ }^{\text {au }}$, L. Bellucci ${ }^{\text {p }}$, J. Berdugo ${ }^{y}$, P. Berges " , B. Bertucci ${ }^{\text {a }}$, B.L. Betev ${ }^{\text {au }}$, S.Bhattacharya ${ }^{j}$, M. Biasini af , A. Biland au, J.J. Blaising ${ }^{d}$, S.C. Blyth ${ }^{\text {ag }}$, G.J. Bobbink ${ }^{\text {b }}$, A. Böhm a , L. Boldizsar ${ }^{\text {m }}$, B. Borgia ${ }^{\text {ai }}$, D. Bourilkov ${ }^{\text {au }}$,

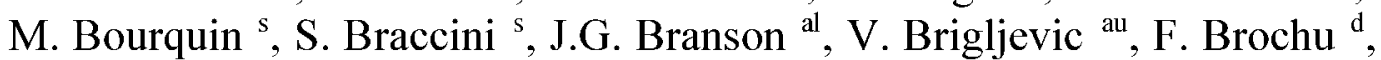
A. Buffini ${ }^{p}$, A. Buijs ${ }^{\text {aq }}$, J.D. Burger ${ }^{n}$, W.J. Burger ${ }^{\text {af }}$, J. Busenitz ${ }^{\text {ap }}$, A. Button ${ }^{c}$, X.D. Cai ", M. Campanelli au, M. Capell n , G. Cara Romeo ', G. Carlino ab, A.M. Cartacci ${ }^{\text {p }}$, J. Casaus ${ }^{y}$, G. Castellini ${ }^{\text {p }, ~ F . ~ C a v a l l a r i ~ a i, ~ N . ~ C a v a l l o ~}{ }^{\text {ab }}$, C. Cecchi s, M. Cerrada ${ }^{y}$, F. Cesaroni w, M. Chamizo ${ }^{\text {s, }}$ Y.H. Chang ${ }^{\text {aw }}$, U.K. Chaturvedi ${ }^{r}$, M. Chemarin ${ }^{x}$, A. Chen ${ }^{\text {aw }}$, G. Chen ${ }^{g}$, G.M. Chen ${ }^{g}$, H.F. Chen ${ }^{\mathrm{t}}$, H.S. Chen ${ }^{\mathrm{g}}$, X. Chereau ${ }^{\mathrm{d}}$, G. Chiefari ${ }^{\mathrm{ab}}$, L. Cifarelli ${ }^{\text {ak }}$, F. Cindolo ${ }^{\mathrm{i}}$,

C. Civinini $^{p}$, I. Clare ${ }^{n}$, R. Clare ${ }^{n}$, G. Coignet ${ }^{d}$, A.P. Colijn ${ }^{b}$, N. Colino ${ }^{y}$, S. Costantini ${ }^{\mathrm{h}}$, F. Cotorobai ${ }^{~}$, B. Cozzoni ${ }^{\mathrm{i}}$, B.de la Cruz ${ }^{\mathrm{y}}$, A. Csilling ${ }^{\mathrm{m}}$, S. Cucciarelli af , T.S. Dai ", J.A. van Dalen ${ }^{\text {ad }}$, R. D'Alessandro ${ }^{\text {p }}$, R. de Asmundis ${ }^{\text {ab }}$, P. Déglon ${ }^{s}$, A. Degré ${ }^{d}, K$. Deiters ${ }^{\text {as }}$, D. della Volpe ${ }^{\text {ab }}$, P. Denes ${ }^{\text {ah }}$, F. DeNotaristefani ${ }^{\text {ai }}$, A. De Salvo ${ }^{\text {au }}, \mathrm{M}$. Diemoz ${ }^{\text {ai }}$, D. van Dierendonck ${ }^{\text {b }}$, F. Di Lodovico au, C. Dionisi ${ }^{\text {ai }}$, M. Dittmar ${ }^{\text {au }}$, A. Dominguez ${ }^{\text {al }}$, A. Doria ${ }^{\text {ab }}$, M.T. Dova ${ }^{r, 1}$, D. Duchesneau ${ }^{d}$, D. Dufournand ${ }^{d}$, P. Duinker ${ }^{b}$, I. Duran ${ }^{\text {am }}$, H. El Mamouni ${ }^{x}$, A. Engler ${ }^{\text {ag }}$, F.J. Eppling ${ }^{n}$, F.C. Erné ${ }^{b}$, P. Extermann ${ }^{\text {s, }}$, M. Fabre ${ }^{\text {as }}$, R. Faccini ${ }^{\text {ai }}$, M.A. Falagan ${ }^{y}$, S. Falciano ${ }^{\text {ai, }}$, A. Favara ${ }^{\mathrm{q}}$, J. Fay ${ }^{\mathrm{x}}$, O. Fedin ${ }^{\text {aj }}$, M. Felcini ${ }^{\text {au }}$, T. Ferguson ${ }^{\text {ag }}$, F. Ferroni ${ }^{\text {ai }}$, H. Fesefeldt ${ }^{\text {a }}$, E. Fiandrini ${ }^{\text {af }}$, J.H. Field s , F. Filthaut ${ }^{\mathrm{q}}$, P.H. Fisher ", I. Fisk ${ }^{\text {al }}$, G. Forconi ", 
L. Fredj s, K. Freudenreich ${ }^{\text {au }}$, C. Furetta ${ }^{z}$, M. Gailloud v, Yu. Galaktionov aa,n, S.N. Ganguli ${ }^{j}$, P. Garcia-Abia ${ }^{e}$, M. Gataullin ${ }^{\text {ae }}$, S.S. Gau ${ }^{k}$, S. Gentile ${ }^{\text {ai, }}$, N. Gheordanescu ${ }^{1}$, S. Giagu ${ }^{\text {ai }}$, S. Goldfarb ${ }^{\text {v }, ~ Z . F . ~ G o n g ~}{ }^{\text {t }}$, G. Grenier ${ }^{\mathrm{x}}$, O. Grimm ${ }^{\text {au }}$, M.W. Gruenewald ${ }^{\text {h }}$, M. Guida ${ }^{\text {ak }}$, R. van Gulik ${ }^{\text {b }, ~ V . K . ~ G u p t a ~}{ }^{\text {ah }}$, A. Gurtu ${ }^{\text {i }}$, L.J. Gutay ${ }^{\text {ar }}$, D. Haas ${ }^{\text {e }}$, A. Hasan ${ }^{\text {ac }}$, D. Hatzifotiadou ${ }^{\mathrm{i}}$, T. Hebbeker ${ }^{\text {h }}$, A. Hervé ${ }^{\mathrm{q}}$, P. Hidas ${ }^{\mathrm{m}}$, J. Hirschfelder ${ }^{\mathrm{ag}}$, H. Hofer ${ }^{\text {au }}$, G. Holzner au ${ }^{\text {au }}$ H. Hoorani ${ }^{\text {ag }}$, S.R. Hou ${ }^{\text {aw }}$, I. Iashvili at , B.N. Jin ${ }^{\mathrm{g}}$, L.W. Jones ${ }^{\text {c }}$, P. de Jong ${ }^{\text {b }}$, I. Josa-Mutuberría ${ }^{\mathrm{y}}$, R.A. Khan ${ }^{\mathrm{r}}$, D. Kamrad ${ }^{\text {at }}$, A. Kasser ${ }^{\mathrm{v}}$, M. Kaur ${ }^{\text {r,2 }}$, M.N. Kienzle-Focacci ${ }^{\text {s }}$, D. Kim ${ }^{\text {ai }}$, D.H. Kim ${ }^{\text {ao }}$, J.K. Kim ${ }^{\text {ao }}$, S.C. Kim ${ }^{\text {ao }}$, J. Kirkby ${ }^{q}$, D. Kiss ${ }^{\text {m}}$, W. Kittel ${ }^{\text {ad }}$, A. Klimentov ${ }^{\text {n,aa }}$, A.C. König ${ }^{\text {ad }}$, A. Kopp ${ }^{\text {at }}$, I. Korolko ${ }^{\text {aa }}$, V. Koutsenko ${ }^{\text {naa }}$, M. Kräber ${ }^{\text {au }}$, R.W. Kraemer ${ }^{\text {ag }}$, W. Krenz a , A. Kunin n,aa , P. Ladron de Guevara ${ }^{\mathrm{y}}$, I. Laktineh ${ }^{\mathrm{x}}$, G. Landi ${ }^{\mathrm{p}}$, K. Lassila-Perini ${ }^{\text {au }}$, P. Laurikainen ${ }^{\text {u }}$, A. Lavorato ${ }^{\text {ak }}$, M. Lebeau ${ }^{\mathrm{q}}$, A. Lebedev ${ }^{\mathrm{n}}$, P. Lebrun ${ }^{x}$, P. Lecomte ${ }^{\text {au }}$, P. Lecoq ${ }^{\mathrm{q}}$, P. Le Coultre ${ }^{\mathrm{au}}$, H.J. Lee ${ }^{\mathrm{h}}$, J.M. Le Goff ${ }^{q}$, R. Leiste ${ }^{\text {at }}$, E. Leonardi ${ }^{\text {ai }}$, P. Levtchenko ${ }^{\text {aj }}$, C. Li ${ }^{\mathrm{t}}$, C.H. Lin ${ }^{\text {aw }}$, W.T. Lin ${ }^{\text {aw }}$, F.L. Linde ${ }^{\text {b }}$, L. Lista ${ }^{\text {ab }}$, Z.A. Liu ${ }^{\mathrm{g}}$, W. Lohmann ${ }^{\text {at }}$, E. Longo ${ }^{\text {ai }}$, Y.S. Lu ${ }^{\text {g }}$, K. Lübelsmeyer ${ }^{\text {a }}$, C. Luci q,ai , D. Luckey ${ }^{n}$, L. Lugnier ${ }^{\text {x }}$, L. Luminari ai, W. Lustermann ${ }^{\text {au }}$, W.G. Ma ${ }^{\mathrm{t}}$, M. Maity ${ }^{\mathrm{j}}$, L. Malgeri ${ }^{\mathrm{q}}$, A. Malinin ${ }^{\mathrm{a}, \mathrm{q}}$, C. Maña ${ }^{\mathrm{y}}$, D. Mangeol ad , P. Marchesini ${ }^{\text {au }}$, G. Marian ${ }^{\circ}$, J.P. Martin ${ }^{x}$, F. Marzano ${ }^{\text {ai }}$, G.G.G. Massaro b , K. Mazumdar ${ }^{\text {j, R.R. McNeil }}{ }^{\text {f }}$, S. Mele ${ }^{\mathrm{q}}$, L. Merola ${ }^{\mathrm{ab}}$, M. Meschini ${ }^{\text {p }}$, W.J. Metzger ad , M. von der Mey a , Y. Mi v, A. Mihul ${ }^{1}$, H. Milcent ${ }^{\mathrm{q}}$, G. Mirabelli ai, J. Mnich ${ }^{\mathrm{q}}$, G.B. Mohanty j, P. Molnar ${ }^{\text {h }}$, B. Monteleoni ${ }^{\text {p,3 }}$, T. Moulik ${ }^{j}$, G.S. Muanza ${ }^{x}$, F. Muheim s, A.J.M. Muijs ${ }^{\text {b }}$, M. Musy ${ }^{\text {ai }}$, M. Napolitano ${ }^{\text {ab }}$, F. Nessi-Tedaldi au, H. Newman ${ }^{\text {ae }}$, T. Niessen ${ }^{\text {a }}$, A. Nippe ${ }^{\mathrm{v}}$, A. Nisati ${ }^{\text {ai }}$, H. Nowak ${ }^{\text {at }}$, Y.D. Oh ${ }^{\text {ao }}$, G. Organtini ai ${ }^{\text {a }}$ R. Ostonen ${ }^{\text {, }}$, C. Palomares ${ }^{y}$, D. Pandoulas ${ }^{\text {a }}$, S. Paoletti ai, ${ }^{\text {, }}$ P. Paolucci ${ }^{\text {ab }}$, R. Paramatti ai, H.K. Park ${ }^{\text {ag }}$, I.H. Park ${ }^{\text {ao }}$, G. Pascale ${ }^{\text {ai }}$, G. Passaleva ${ }^{\text {q }}$, S. Patricelli ab, T. Paul ${ }^{\text {k, }}$, M. Pauluzzi ${ }^{\text {af }}$, C. Paus ${ }^{q}$, F. Pauss ${ }^{\text {au }}$, D. Peach ${ }^{\text {q }}$, M. Pedace ${ }^{\text {ai }}$, S. Pensotti ${ }^{z}$, D. Perret-Gallix ${ }^{\mathrm{d}}$, B. Petersen ${ }^{\text {ad }}$, D. Piccolo ${ }^{\mathrm{ab}}$, F. Pierella ${ }^{\mathrm{i}}$, M. Pieri ${ }^{\mathrm{p}}$, P.A. Piroué ah ${ }^{\text {, E. Pistolesi }}{ }^{\text {, }}$, V. Plyaskin ${ }^{\text {aa }}$, M. Pohl au ${ }^{\text {, V. Pojidaev }}{ }^{\text {aa, }}$, H. Postema ${ }^{\text {, }}$ J. Pothier ${ }^{\mathrm{q}}$, N. Produit s, D.O. Prokofiev ${ }^{\text {ar }}$, D. Prokofiev aj, J. Quartieri ak, G. Rahal-Callot ${ }^{a u, q}$, M.A. Rahaman ${ }^{\mathrm{j}}$, P. Raics ${ }^{\circ}$, N. Raja ${ }^{\mathrm{j}}$, R. Ramelli ${ }^{\text {au }}$, P.G. Rancoita ${ }^{z}$, G. Raven ${ }^{\text {al }}$, P. Razis ${ }^{\text {ac }}$, D. Ren ${ }^{\text {au }}$, M. Rescigno ${ }^{\text {ai }}$, S. Reucroft ${ }^{k}$, T. van Rhee ${ }^{\text {aq }}$, S. Riemann ${ }^{\text {at }}$, K. Riles ${ }^{\text {c }}$, A. Robohm ${ }^{\text {au }}$, J. Rodin ${ }^{\text {ap }}$, B.P. Roe ${ }^{\text {c }}$, L. Romero ${ }^{\mathrm{y}}$, A. Rosca ${ }^{\mathrm{h}}$, S. Rosier-Lees ${ }^{\text {d, Ph. Rosselet }}{ }^{\text {v }}$, J.A. Rubio ${ }^{\mathrm{q}}$, D. Ruschmeier ${ }^{\text {h }}$, H. Rykaczewski ${ }^{\text {au }}$, S. Sarkar ai, J. Salicio ${ }^{q}$, E. Sanchez ${ }^{\text {q }}$, M.P. Sanders ${ }^{\text {ad }}$, M.E. Sarakinos ${ }^{\text {u }}$, C. Schäfer ${ }^{\text {a }}$, V. Schegelsky ${ }^{\text {aj, }}$ S. Schmidt-Kaerst a , D. Schmitz a , H. Schopper ${ }^{\text {av }}$, D.J. Schotanus ${ }^{\text {ad }}$, G. Schwering a , 
C. Sciacca ${ }^{\text {ab }}$, D. Sciarrino ${ }^{\text {s, }}$ A. Seganti ${ }^{\text {i }}$, L. Servoli af, S. Shevchenko ${ }^{\text {ae }}$, N. Shivarov an, V. Shoutko aa, E. Shumilov aa , A. Shvorob ${ }^{\text {ae }}$, T. Siedenburg a , D. Son ${ }^{\text {ao }}$, B. Smith ${ }^{\text {ag }}$, P. Spillantini ${ }^{p}$, M. Steuer ${ }^{n}$, D.P. Stickland ${ }^{\text {ah }}$, A. Stone ${ }^{\text {f }}$, H. Stone ah,3 B. Stoyanov an, A. Straessner a, K. Sudhakar ${ }^{j}$, G. Sultanov ${ }^{r}$, L.Z. Sun ${ }^{\mathrm{t}}$, H. Suter ${ }^{\text {au }}$, J.D. Swain ${ }^{\mathrm{r}}$, Z. Szillasi ap,4, T. Sztaricshai ${ }^{\text {ap, }}{ }^{4}$, X.W. Tang ${ }^{\mathrm{g}}$, L. Tauscher ${ }^{\mathrm{e}}$, L. Taylor ${ }^{\mathrm{k}}$, C. Timmermans ${ }^{\mathrm{ad}}$, Samuel C.C. Ting ${ }^{n}$, S.M. Ting ${ }^{n}$, S.C. Tonwar ${ }^{j}$, J. Tóth ${ }^{\mathrm{m}}$, C. Tully ${ }^{\text {ah }}$, K.L. Tung ${ }^{\mathrm{g}}$, Y. Uchida ${ }^{\mathrm{n}}$, J. Ulbricht ${ }^{\text {au }}$, E. Valente ${ }^{\text {ai }}$, G. Vesztergombi ${ }^{\mathrm{m}}$, I. Vetlitsky ${ }^{\text {aa }}$, D. Vicinanza ${ }^{\text {ak }}$, G. Viertel ${ }^{\text {au }}$, S. Villa ${ }^{k}$, M. Vivargent ${ }^{d}$, S. Vlachos ${ }^{e}$, I. Vodopianov aj, H. Vogel ag, H. Vogt ${ }^{\text {at }}$, I. Vorobiev ${ }^{\text {aa }}$, A.A. Vorobyov ${ }^{\text {aj, }}$, A. Vorvolakos ${ }^{\text {ac }}$, M. Wadhwa ${ }^{e}$, W. Wallraff a, M. Wang " , X.L. Wang ", Z.M. Wang ${ }^{\mathrm{t}}$, A. Weber ${ }^{\mathrm{a}}$, M. Weber a, P. Wienemann a , H. Wilkens ad, S.X. Wu ${ }^{n}$, S. Wynhoff ${ }^{a}$, L. Xia ${ }^{\text {ae }}$, Z.Z. Xu ${ }^{\mathrm{t}}$, B.Z. Yang ${ }^{\mathrm{t}}$, C.G. Yang ${ }^{\mathrm{g}}$, H.J. Yang ${ }^{\mathrm{g}}$, M. Yang ${ }^{\mathrm{g}}$, J.B. Ye ${ }^{\mathrm{t}}$, S.C. Yeh ${ }^{\text {ax }}$, An. Zalite ${ }^{\text {aj }}$, Yu. Zalite ${ }^{\text {aj }}$, Z.P. Zhang ${ }^{\mathrm{t}}$, G.Y. Zhu ${ }^{\mathrm{g}}$, R.Y. Zhu ${ }^{\text {ae }}$, A. Zichichi i, ${ }^{\text {, }, \mathrm{r}}$, F. Ziegler ${ }^{\text {at }}$, G. Zilizi ${ }^{\text {ap, }}{ }^{\text {, }}$ M. Zöller ${ }^{\text {a }}$

\author{
${ }^{a}$ I. Physikalisches Institut, RWTH, D-52056 Aachen, Germany, \\ and III. Physikalisches Institut, RWTH, D-52056 Aachen, Germany \\ ${ }^{\mathrm{b}}$ National Institute for High Energy Physics, NIKHEF, and University of Amsterdam, NL-1009 DB Amsterdam, The Netherlands \\ ${ }^{c}$ University of Michigan, Ann Arbor, $M I$ 48109, USA \\ ${ }^{d}$ Laboratoire d'Annecy-le-Vieux de Physique des Particules, LAPP, IN2P3-CNRS, BP 110, F-74941 Annecy-le-Vieux CEDEX, France \\ ${ }^{\mathrm{e}}$ Institute of Physics, University of Basel, $\mathrm{CH}-4056$ Basel, Switzerland \\ ${ }^{\mathrm{f}}$ Louisiana State University, Baton Rouge, LA 70803, USA \\ ${ }^{g}$ Institute of High Energy Physics, IHEP, 100039 Beijing, China ${ }^{6}$ \\ ${ }^{\mathrm{h}}$ Humboldt University, D-10099 Berlin, Germany ${ }^{5}$ \\ ${ }^{\mathrm{i}}$ University of Bologna and INFN-Sezione di Bologna, I-40126 Bologna, Italy \\ ${ }^{\mathrm{j}}$ Tata Institute of Fundamental Research, Bombay 400005 , India \\ ${ }^{\mathrm{k}}$ Northeastern University, Boston, MA 02115, USA \\ ${ }^{1}$ Institute of Atomic Physics and University of Bucharest, $R-76900$ Bucharest, Romania \\ ${ }^{\mathrm{m}}$ Central Research Institute for Physics of the Hungarian Academy of Sciences, H-1525 Budapest 114, Hungary ${ }^{7}$ \\ ${ }^{\mathrm{n}}$ Massachusetts Institute of Technology, Cambridge, MA 02139, USA \\ ${ }^{\circ}$ Lajos Kossuth University-ATOMKI, H-4010 Debrecen, Hungary ${ }^{4}$ \\ ${ }^{\mathrm{P}}$ INFN Sezione di Firenze and University of Florence, I-50125 Florence, Italy \\ ${ }^{\mathrm{q}}$ European Laboratory for Particle Physics, CERN, CH-1211 Geneva 23, Switzerland \\ ${ }^{\mathrm{r}}$ World Laboratory, FBLJA Project, CH-1211 Geneva 23, Switzerland \\ s University of Geneva, $\mathrm{CH}-1211$ Geneva 4, Switzerland \\ ${ }^{\mathrm{t}}$ Chinese University of Science and Technology, USTC, Hefei, Anhui 230 029, China ${ }^{6}$ \\ ${ }^{\mathrm{u}}$ SEFT, Research Institute for High Energy Physics, P.O. Box 9, SF-00014 Helsinki, Finland \\ 'University of Lausanne, $\mathrm{CH}-1015$ Lausanne, Switzerland \\ ${ }^{w}$ INFN-Sezione di Lecce and Universitá Degli Studi di Lecce, I-73100 Lecce, Italy \\ ${ }^{\mathrm{x}}$ Institut de Physique Nucléaire de Lyon, IN2P3-CNRS, Université Claude Bernard, F-69622 Villeurbanne, France \\ ${ }^{\text {y }}$ Centro de Investigaciones Energéticas, Medioambientales y Tecnologicas, CIEMAT, E-28040 Madrid, Spain ${ }^{8}$ \\ ${ }^{\mathrm{z}}$ INFN-Sezione di Milano, I-20133 Milan, Italy \\ anstitute of Theoretical and Experimental Physics, ITEP, Moscow, Russia \\ ${ }^{\mathrm{ab}}$ INFN-Sezione di Napoli and University of Naples, I-80125 Naples, Italy

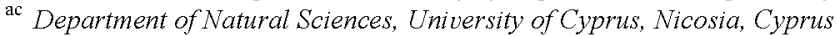 \\ ${ }^{\text {ad }}$ University of Nijmegen and NIKHEF, NL-6525 ED Nijmegen, The Netherlands \\ ae California Institute of Technology, Pasadena, CA 91125, USA \\ af INFN-Sezione di Perugia and Universitá Degli Studi di Perugia, I-06100 Perugia, Italy \\ ag Carnegie Mellon University, Pittsburgh, PA 15213, USA
}




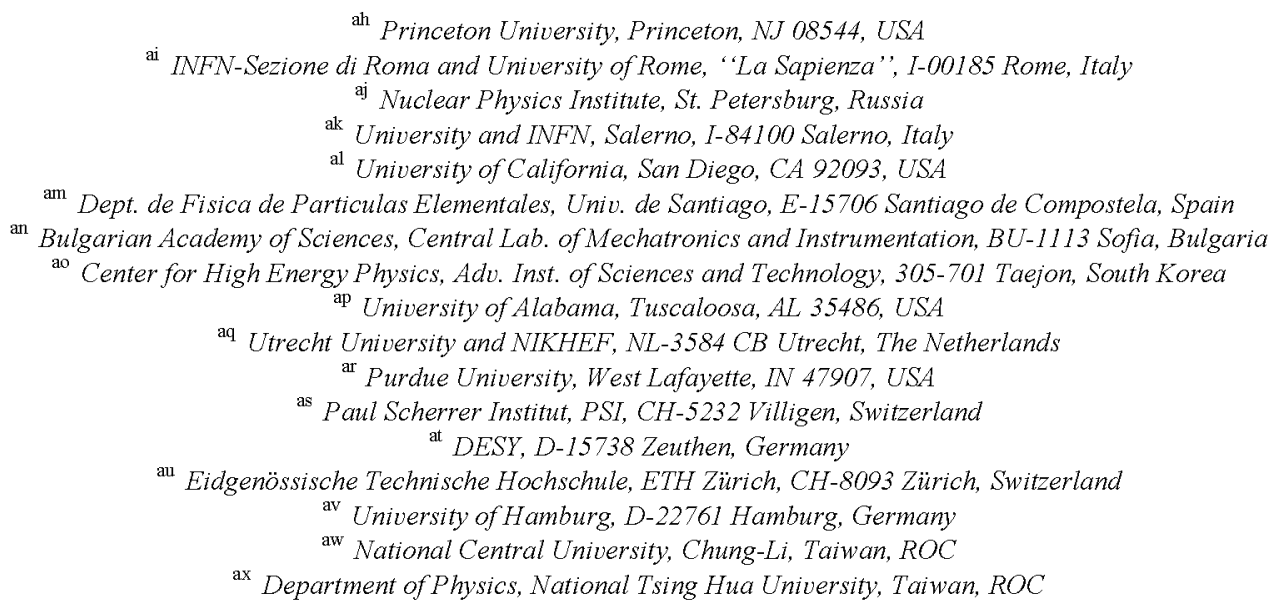

Received 6 August 1999; accepted 10 September 1999

Editor: K. Winter

\begin{abstract}
We measure the masses, decay widths and relative production rate of orbitally excited B mesons using 1.25 million hadronic $Z$ decays recorded by the L3 detector. B-meson candidates are inclusively reconstructed and combined with charged pions produced at the primary event vertex. An excess of events above the expected background in the $\mathrm{B} \pi$ mass spectrum in the region $5.6-5.8 \mathrm{GeV}$ is interpreted as resulting from the decay $\mathrm{B}_{\mathrm{u}, \mathrm{d}}^{* *} \rightarrow \mathrm{B}^{(*)} \pi$, where $\mathrm{B}_{\mathrm{u}, \mathrm{d}}^{*}$ denotes a mixture of $l=1 \mathrm{~B}-$ meson states containing a $\mathrm{u}$ or a d quark. A fit to the mass spectrum yields the masses and decay widths of the $\mathrm{B}_{1}^{*}$ and $\mathrm{B}_{2}^{*}$ spin states, as well as the branching fraction for the combination of $l=1$ states. In addition, evidence is presented for the existence of an excited B-meson state or mixture of states in the region 5.9-6.0 GeV. C 1999 Published by Elsevier Science B.V. All rights reserved.
\end{abstract}

\footnotetext{
${ }^{1}$ Also supported by CONICET and Universidad Nacional de La Plata, CC 67, 1900 La Plata, Argentina.

${ }^{2}$ Also supported by Panjab University, Chandigarh-160014, India.

${ }^{3}$ Deceased.

${ }^{4}$ Also supported by the Hungarian OTKA fund under contract numbers T22238 and T026178.

${ }^{5}$ Supported by the German Bundesministerium für Bildung, Wissenschaft, Forschung und Technologie.

${ }^{6}$ Supported by the National Natural Science Foundation of China.

${ }^{7}$ Supported by the Hungarian OTKA fund under contract numbers T019181, F023259 and T024011.

${ }^{8}$ Supported also by the Comisión Interministerial de Ciencia y Tecnología.
}

\section{Introduction}

The spectroscopy of orbitally excited B mesons provides important information regarding the underlying QCD potential. A flavor-spin symmetry [1] arises from the fact that the mass of the $b$ quark is large relative to $\Lambda_{\mathrm{QCD}}$. In this approximation, the spin $\left(s_{\mathrm{Q}}\right)$ of the heavy quark $(\mathrm{Q})$ is conserved in production and decay processes independently of the total angular momentum $\left(j_{\mathrm{q}}=\boldsymbol{s}_{\mathrm{q}}+\boldsymbol{l}\right)$ of the light quark (q). Excitation energy levels are thus degenerate doublets in total spin and can be expressed in terms of the spin-parity of the meson, $J^{P}$, and the total spin of the light quark, $j_{\mathrm{q}}$. The $l=0$ mesons, for which $j_{\mathrm{q}}=1 / 2$, have two possible spin states: a pseudo-scalar $P$, corresponding to $J^{P}=0^{-}$, and a vector $V$, corresponding to $J^{P}=1^{-}$. If the spin of 
the heavy quark is independently conserved, the relative production rate of these states would be $V /(V+P)=0.75^{9}$. Recent measurements of the $\mathrm{B}^{*}$ production rate using $\mathrm{B}^{*} \rightarrow \mathrm{B} \gamma$ decays in $\mathrm{e}^{+} \mathrm{e}^{-}$ collisions [2,3] agree well with this number.

For orbitally excited mesons with $l=1$, there are two sets of degenerate doublets: one corresponding to $j_{\mathrm{q}}=1 / 2$ and the other to $j_{\mathrm{q}}=3 / 2$. For the $j_{\mathrm{q}}=1 / 2$ doublet, there is one state corresponding to $J^{P}=0^{+}$and three degenerate states corresponding to $J^{P}=1^{+}$. For the $j_{\mathrm{q}}=3 / 2$ doublet, there are three degenerate states corresponding to $J^{P}=1^{+}$and five others corresponding to $J^{P}=2^{+}$. Rules for the decay of the $l=1$ states to the $l=0$ states are dictated by spin-parity conservation [4]. For the dominant two-body decays to a pion and a $\mathrm{B}$ meson, the $j_{\mathrm{q}}=1 / 2$ states undergo an $L=0$ transition (S-wave) and their decay widths are expected to be broad in comparison to those of the $j_{\mathrm{q}}=3 / 2$ states which undergo an $L=2$ transition (D-wave). Table 1 presents the nomenclature of the $l=1 \mathrm{~B}$ mesons containing either $\mathrm{a} u$ or a $\mathrm{d}$ quark, $\mathrm{B}_{\mathrm{u}, \mathrm{d}}^{* *}$, with the corresponding spin states, degeneracies and two-body decay modes. The spectroscopy of $l=1 \mathrm{~B}$ mesons containing an $s$ quark is not studied in this analysis, but is examined as a possible source of systematic uncertainty.

Predictions for the masses and decay widths of the four spin states are based on Heavy Quark Effective Theory (HQET), in which corrections to the spectator quark model are expressed as perturbations in powers of $\Lambda_{\mathrm{QCD}} / m_{\mathrm{Q}}$ [5-12]. Such corrections, which can be relatively large for c hadrons, are considerably smaller for hadrons containing the more massive b quark.

Recent analyses at LEP, in which a charged pion produced at the primary event vertex is combined with an inclusively reconstructed $B$ meson [13], have measured an average $B_{u, d}^{* *}$ mass in the range 5700$5730 \mathrm{MeV}$. An analysis combining a primary charged pion with a fully reconstructed $B$ meson [14] measures $M_{\mathrm{B}_{2}^{*}}=\left(5739_{-11}^{+8}\right.$ (stat) ${ }_{-4}^{+6}$ (syst) $) \mathrm{MeV}$ by performing a fit to the mass spectrum which fixes the

\footnotetext{
${ }^{9}$ Corrections due to the decay of higher excited states are predicted to be small.
}

Table 1

Spin states of the $l=1 \mathrm{~B}_{\mathrm{u}, \mathrm{d}}$ mesons with the expected relative production rates according to spin counting $(2 J+1)$ and the associated decay modes and transitions predicted by spin-parity conservation.

\begin{tabular}{lllll}
\hline$j_{\mathrm{q}}$ & $J^{P}$ & $2 J+1$ & Decay mode & Transition \\
\hline $1 / 2$ & $0^{+}$ & 1 & $\mathrm{~B}_{0}^{*} \rightarrow \mathrm{B} \pi$ & S-wave \\
$1 / 2$ & $1^{+}$ & 3 & $\mathrm{~B}_{1}^{*} \rightarrow \mathrm{B}^{*} \pi$ & S-wave \\
$3 / 2$ & $1^{+}$ & 3 & $\mathrm{~B}_{1} \rightarrow \mathrm{B}^{*} \pi$ & D-wave \\
$3 / 2$ & $2^{+}$ & 5 & $\mathrm{~B}_{2}^{*} \rightarrow \mathrm{B}^{*} \pi, \mathrm{B} \pi$ & D-wave \\
\hline
\end{tabular}

mass differences, widths and relative rates of all spin states according to the predictions of Ref. [8].

The analysis presented here is based on combining a primary charged pion with an inclusively reconstructed B meson. We use new techniques both to improve the resolution of the reconstructed $\mathrm{B} \pi$ mass spectrum and to unfold this resolution from the signal components. As a result, we are able to extract measurements for the masses and widths of both the $\mathrm{S}$-wave $\mathrm{B}_{1}^{*}$ decays and the $\mathrm{D}$-wave $\mathrm{B}_{2}^{*}$ decays.

\section{Event selection and reconstruction}

\subsection{Selection of $Z \rightarrow \bar{b} \bar{b}$ decays}

The data, collected by the L3 detector [15] in 1994 and 1995, correspond to an integrated luminosity of $90 \mathrm{pb}^{-1}$ with centre-of-mass energies around the $Z$ mass. Hadronic $Z$ decays are selected by making use of their characteristic energy distributions and high multiplicity [16]. In addition, all events are required to have an event thrust axis direction satisfying $|\cos \theta|<0.74$, where $\theta$ is the polar angle; to contain a primary vertex reconstructed in three dimensions; to contain at least two calorimetric jets, each with energy greater than $10 \mathrm{GeV}$; and to pass stringent detector quality criteria for the vertexing, tracking and calorimetry. A total of 1248350 events pass this selection. Cutting on $\mathrm{a} Z \rightarrow \mathrm{b} \overline{\mathrm{b}}$ event discriminant based on the lifetime information of charged constituents [17] yields a b-enriched sample of 176980 events.

A sample of 6 million simulated hadronic $Z$ decays have been generated with JETSET 7.4 [18] and passed through the L3 simulation program [19] to 
study the content of the selected data. From this study, the purity of the $Z \rightarrow b \bar{b}$ candidates is determined to be $83 \%$ and corresponds to a selection efficiency of $65 \%$.

\subsection{Selection and reconstruction of $B^{* *} \rightarrow B^{(*)} \pi$ decays}

The primary event vertex and secondary decay vertices are reconstructed in three dimensions on an event-by-event basis such that each charged track can be a constituent of no more than one vertex. A calorimetric jet is selected for analysis as a B candidate if it is one of the two most energetic jets in the event, if a secondary decay vertex is reconstructed from tracks associated with that jet, and if the distance of that vertex with respect to the primary event vertex is greater than three times the estimated error of the measurement.

The decay $\mathrm{B}_{\mathrm{u}, \mathrm{d}}^{* *} \rightarrow \mathrm{B}^{(*)} \pi$ is a strong interaction and thus occurs at the primary event vertex. In addition, the predicted masses for the $l=1$ states correspond to relatively small $Q$ values, so that the decay pion direction tends to be forward with respect to the B-meson direction (Fig. 1). We take advantage of these decay kinematics by requiring that, for each B-meson candidate, there be at least one track which originates from the primary event vertex and which is located in the hemisphere defined by the jet thrust axis direction. No attempt is made to identify the

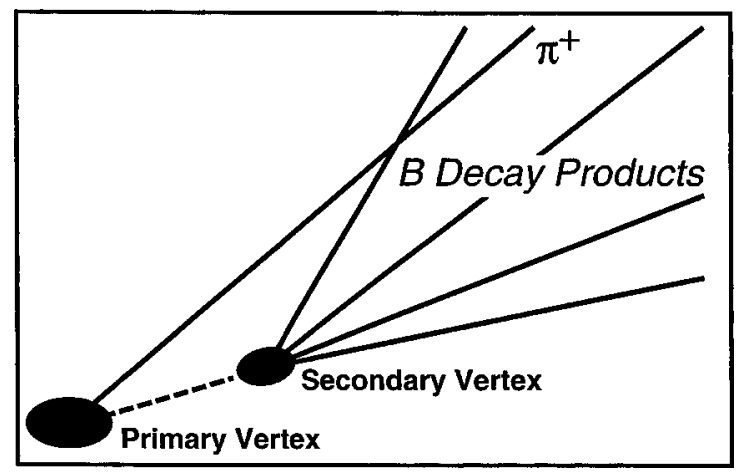

Fig. 1. Schematic of the decay $\mathrm{B}^{* *} \rightarrow \mathrm{B}^{(*)} \pi$. Ellipses represent the reconstruction uncertainties of the vertex positions. The decay pion points to the primary vertex and its direction is forward with respect to the direction of the $B$ meson. track as a pion. A total of 60205 track-jet pairs satisfy these criteria.

To further decrease background, which is typically due to charged particles from fragmentation, only the track with the largest component of momentum along the direction of the jet is selected. This method has been found $[20,14]$ to be an efficient means to improve the purity of the signal. In addition, background due to charged pions from $\mathrm{D}^{*} \rightarrow$ $\mathrm{D} \pi$ decays is reduced by requiring the track to have a transverse momentum with respect to the jet axis larger than $100 \mathrm{MeV}$. These selection criteria are satisfied by 48022 track-jet pairs with a b-hadron purity of $94.2 \%$.

The direction of the $\mathrm{B}$ candidate is estimated by taking an error-weighted average in the $\theta$ (polar) and $\phi$ (azimuthal) coordinates of the directions defined by the vertices and by particles with a high rapidity relative to the jet axis. A numerical error-propagation method [21] makes it possible to obtain accurate estimates for the uncertainty of the angular coordinates measured from vertex pairs. These errors, as well as the error for the decay length measurement used in the secondary vertex selection, are calculated for each pair of vertices from the associated error matrices and determine the weight for the vertex-defined coordinate measurements.

A second estimate for the direction of the $B$ meson is obtained by summing the momenta of all charged and neutral particles (excluding the decay pion candidate) with rapidity $y>1.6$ relative to the jet axis. Such particles have a high probability to be decay products of the $\mathrm{B}^{(*)}$ meson. Estimates for the uncertainty of these coordinates are determined from simulated B-meson decays as an average value for all events and determine the weight for the rapiditydefined coordinate measurements. The final B-meson direction coordinates are taken as the error-weighted averages of the two sets of coordinates.

The resolution for each coordinate is parametrized by a two-Gaussian fit to the difference between the reconstructed and generated values. For $\theta$, the two widths are $\sigma_{1}=18 \mathrm{mrad}$ and $\sigma_{2}=34 \mathrm{mrad}$, with $68 \%$ of the B mesons in the first Gaussian. For $\phi$, the two widths are $\sigma_{1}=12 \mathrm{mrad}$ and $\sigma_{2}=34 \mathrm{mrad}$, with $62 \%$ of the B mesons in the first Gaussian.

The energy of the B-meson candidate is estimated by taking advantage of the known centre-of-mass 
energy at LEP to constrain the measured value. The energy of the B meson from this method can be expressed as

$E_{\mathrm{B}}=\frac{E_{\mathrm{cm}}^{2}-M_{\mathrm{recoil}}^{2}+M_{\mathrm{B}}^{2}}{2 E_{\mathrm{cm}}}$,

where $E_{\mathrm{cm}}$ is the centre-of-mass energy, $M_{\text {recoil }}$ is the mass of all particles in the event with rapidity $y<1.6$, including the decay pion candidate (regardless of its rapidity) and $M_{\mathrm{B}}$ is the known B-meson mass. The resolution is estimated by the difference between reconstructed and generated B-meson energy values; it is best described by a bifurcated Gaussian [22] with a lower width of $1.9 \mathrm{GeV}$ and an upper width of $2.8 \mathrm{GeV}$.

\section{Analysis of the $B \pi$ mass spectrum}

The $\mathrm{B} \pi$ mass is calculated as

$M_{\mathrm{B} \pi}=\sqrt{M_{\mathrm{B}}^{2}+m_{\pi}^{2}+2 E_{\mathrm{B}} E_{\pi}-2 p_{\mathrm{B}} p_{\pi} \cos \alpha}$,

where $M_{\mathrm{B}}$ and $m_{\pi}$ are, respectively, the known B-meson and charged pion masses, $E_{\mathrm{B}}$ is the B-meson energy described in the previous section, $p_{\pi}$ is the measured pion momentum and $\alpha$ is the measured angle between the B-meson candidate and the decay-pion candidate. The resulting mass spectrum is presented in Fig. 2a, along with the Monte Carlo background, normalized to the region $6.0-6.6 \mathrm{GeV}$.

The background $\mathrm{B} \pi$ mass distribution is estimated from the Monte Carlo sample, excluding $\mathrm{B}^{*}$ * $\rightarrow \mathrm{B}^{(*)} \pi$ decays, and fit with a six-parameter threshold function given by

$$
\begin{aligned}
& p_{1}\left(M_{\mathrm{B} \pi}-p_{2}\right)^{p_{3}} \\
& \quad \times e^{p_{4}\left(M_{\mathrm{B} \pi}-p_{2}\right)+p_{5}\left(M_{\mathrm{B} \pi}-p_{2}\right)^{2}+p_{6}\left(M_{\mathrm{B} \pi}-p_{2}\right)^{3}} .
\end{aligned}
$$

The shape parameters, $p_{2}$ through $p_{6}$, are fixed by the fit to the simulated background, while the overall normalization factor, $p_{1}$, is unconstrained in the fit to the data spectrum. In this manner, the normalization uncertainty is accounted for in the statistical error. The uncertainty due to the background shape is accounted for in the systematic error estimate.

To examine the underlying structure, it is necessary to account for effects due to detector resolution.
The momentum resolution of the decay-pion candidates, which have typical momenta of $1-3 \mathrm{GeV}$, is a few percent, and the angular resolution is better than $2 \mathrm{mrad}$. In this case, the dominant sources of uncertainty for the mass measurement are the B-meson angular and energy resolutions. Monte Carlo studies confirm that these two components of the mass uncertainty are dominant and approximately equal in magnitude. The following analysis parametrizes the effects of these components on the measured $B \pi$ mass and then uses the parametrization to fold the resolution effects into the fitting function.
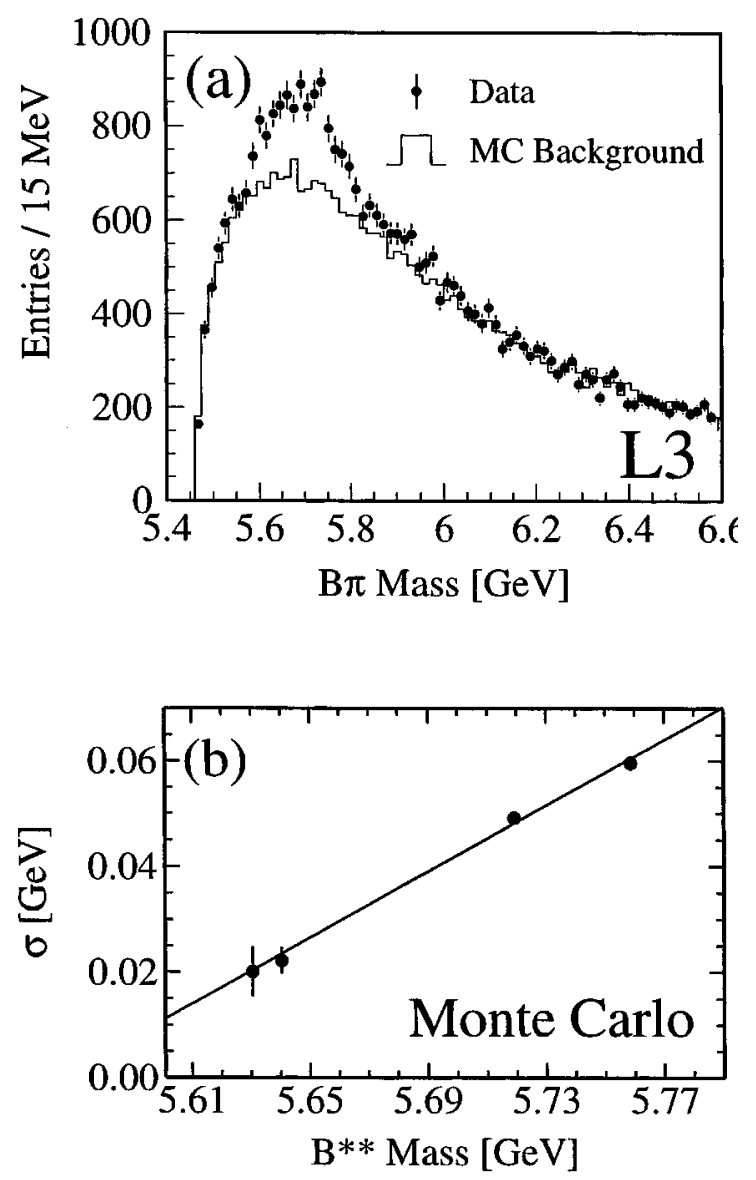

Fig. 2. (a) Mass spectrum for selected $B \pi$ pairs. The points with error bars are data and the histogram represents the expected background from Monte Carlo, normalized to the sideband region 6.0-6.6 GeV. (b) Linear fit of the extracted $\mathrm{B} \pi$ mass resolution for the Monte Carlo signal components at the generated $\mathrm{B}^{* *}$ mass values. 
The dependence of the $\mathrm{B} \pi$ mass resolution on the $\mathrm{B}^{* *}$ mass is studied by simulating $\mathrm{B}^{* *}$ decays at four different values of mass and width. Each $B \pi$ mass distribution is fit using a Voigt function, which is a Breit-Wigner function convoluted with a Gaussian resolution function. The Breit-Wigner width is fixed to its generated value and its mass, normalization and Gaussian resolution are extracted from the fit.

Each extracted $\mathrm{B} \pi$ mass value is found to agree with its generated mass within the statistical error of the fit, and the corresponding efficiencies are found to have no mass dependence. The Gaussian resolution (Fig. 2b) is plotted for each generated $\mathrm{B}^{* *}$ mass value, and fit with a linear function. This function is used to estimate the detector resolution at the measured $\mathrm{B} \pi$ mass.

Agreement between data and Monte Carlo for the B-meson energy and angular resolution is confirmed by analyzing $\mathrm{B}^{*} \rightarrow \mathrm{B} \gamma$ decays selected from the same sample of B mesons. The photon selection for this test is the same as that described in Ref. [2]. A $\mathrm{B}^{*}$ meson decays electromagnetically and thus has a negligible decay width compared to the detector resolution. As in the case of the $\mathrm{B} \pi$ mass resolution, the B-meson energy and angular resolution are the dominant components of the reconstructed $\mathrm{B} \gamma$ mass resolution. Fits to the $M_{\mathrm{B} \gamma}-M_{\mathrm{B}}$ spectra are performed with the combination of a Gaussian signal and the background function described above. For simulated events, the Gaussian mean value is found to be $M_{\mathrm{B} \gamma}-M_{\mathrm{B}}=(46.5 \pm 0.6) \mathrm{MeV}$ with a width of $(11.1 \pm 0.7) \mathrm{MeV}$ for an input generator mass difference of $46.0 \mathrm{MeV}$. For data, the Gaussian mean value is found to be $M_{\mathrm{B} \gamma}-M_{\mathrm{B}}=(45.1 \pm 0.6) \mathrm{MeV}$ with a width of $(10.7 \pm 0.6) \mathrm{MeV}$. Good agreement between the widths of the data and Monte Carlo signals confirms that the B-meson energy and angular resolutions are well understood and simulated.

According to spin-parity conservation, one expects mass peaks from five possible $B_{\text {u,d }}^{*}$ decay modes: $\mathrm{B}_{0}^{*} \rightarrow \mathrm{B} \pi, \mathrm{B}_{1}^{*} \rightarrow \mathrm{B}^{*} \pi, \mathrm{B}_{1} \rightarrow \mathrm{B}^{*} \pi, \mathrm{B}_{2}^{*} \rightarrow$ $\mathrm{B}^{*} \pi$ and $\mathrm{B}_{2}^{*} \rightarrow \mathrm{B} \pi$. No attempt is made to tag subsequent $\mathrm{B}^{*} \rightarrow \mathrm{B} \gamma$ decays, as the efficiency for selecting the soft photon is low. As a consequence, the effective $\mathrm{B} \pi$ masses for the three decays to $\mathrm{B}^{*}$ mesons are shifted down by the $M_{\mathrm{B}}{ }^{*}-M_{\mathrm{B}}=$ $46 \mathrm{MeV}$ mass difference. The distribution is fit with
Table 2

Constraints applied to the relative production rates, masses and widths of the four $B_{u, d}^{* *}$ spin states in the $B \pi$ mass spectrum fit.

\begin{tabular}{lllll}
\hline$j_{\mathrm{q}}$ & Spin state & Production & Mass & Width \\
\hline $1 / 2$ & $\mathrm{~B}_{0}^{*}$ & $1 / 12$ & $M_{\mathrm{B}_{1}^{*}}-12 \mathrm{MeV}$ & $\Gamma_{\mathrm{B}_{1}^{*}}$ \\
$1 / 2$ & $\mathrm{~B}_{1}^{*}$ & $3 / 12$ & free & free \\
$3 / 2$ & $\mathrm{~B}_{1}$ & $3 / 12$ & $M_{\mathrm{B}_{2}^{*}}-12 \mathrm{MeV}$ & $\Gamma_{\mathrm{B}_{2}^{*}}$ \\
$3 / 2$ & $\mathrm{~B}_{2}^{*}$ & $5 / 12$ & free & free \\
\hline
\end{tabular}

five Voigt functions, with the relative production fractions determined by spin counting rules. Gaussian convolution of the decay widths is taken from the resolution function at each measured mass value.

Additional physical constraints, presented in Table 2 , are based on predictions common to existing HQET models [6-12]. In general, these models predict the mass differences for the two $j_{\mathrm{q}}=1 / 2$ states and for the two $j_{q}=3 / 2$ states to be approximately equal and in the range 5-20 MeV. Several of the models [7,9] place the average mass of the $j_{\mathrm{q}}=3 / 2$ states above that of the $j_{\mathrm{q}}=1 / 2$ states, while others $[11,12]$ predict the opposite "spin-orbit inversion." We constrain $M_{\mathrm{B}_{1}^{*}}-M_{\mathrm{B}_{0}^{*}}=12 \mathrm{MeV}$ and $M_{\mathrm{B}_{2}^{*}}-$ $M_{\mathrm{B}_{1}}=12 \mathrm{MeV}$, but allow the masses of the $\mathrm{B}_{1}^{*}$ and $\mathrm{B}_{2}^{*}$ to be free to test the two opposing hypotheses.

Predictions are made for the widths of the $j_{\mathrm{q}}=$ $3 / 2$ states by extrapolating from measurements in the $\mathrm{D}$ meson system [23]. They are expected to be approximately equal and about $20-25 \mathrm{MeV}$. No precise predictions exist for the widths of the $j_{\mathrm{q}}=$ $1 / 2$ states as there are no corresponding measurements in the D system. In general, however, they are also expected to be approximately equal, although broader than those of the $j_{\mathrm{q}}=3 / 2$ states. We constrain $\Gamma_{\mathrm{B}_{0}^{*}}=\Gamma_{\mathrm{B}_{1}^{*}}$ and $\Gamma_{\mathrm{B}_{1}}=\Gamma_{\mathrm{B}_{2}^{*}}$, but allow the widths of the $\mathrm{B}_{1}^{*}$ and $\mathrm{B}_{2}^{*}$ to be free in the fit.

Simulated signal and background mass spectra are combined and then fit with the functions and constraints described above. Mass values and decay widths for the $B_{2}^{*}$ and $B_{1}^{*}$ resonances and the overall normalization are extracted from the fit and found to agree well with the generated values. All differences lie within the statistical errors.

\section{Fit results}

The $\mathrm{B} \pi$ mass spectrum for data is fit with the functions and constraints described above. A total of 
$2770 \pm 394$ events are in the signal region, and the masses and widths of the $B_{1}^{*}$ and $B_{2}^{*}$ mesons are found to be

$M_{\mathrm{B}_{1}^{*}}=(5682 \pm 23$ (stat) $) \mathrm{MeV}$,

$\Gamma_{\mathrm{B}_{1}^{*}}=(73 \pm 44$ (stat) $) \mathrm{MeV}$,

$M_{\mathrm{B}_{2}^{*}}=(5771 \pm 7$ (stat) $) \mathrm{MeV}$,

$\Gamma_{\mathrm{B}_{2}^{*}}=(41 \pm 43$ (stat) $) \mathrm{MeV}$.

The fit has a $\chi^{2}$ of 81 for 70 degrees of freedom. It does not describe the data well in the region 5.9-6.0 $\mathrm{GeV}$, where there appears to be an excess of data events over the simulated background. Exclusion of this region from the fit yields consistent values for the signal and reduces the $\chi^{2}$ to 57 for 60 degrees of freedom.

Several HQET models $[7,12,9]$ predict the existence of radially excited $(2 S)$ B-meson states in the region 5.9-6.0 GeV. In addition, an inclusive measurement of $B \pi \pi$ final states in $Z$ decays [24] provides evidence for a resonance in the same mass region. To account for the possible existence of these states in the mass spectrum, we refit the data including a Gaussian function in the region of interest.

The resulting fit, shown in Fig. 3, has a $\chi^{2}$ of 63 for 67 degrees of freedom. A total of $2784 \pm 274$ events are in the original signal region, corresponding to the branching fraction $\mathrm{Br}\left(\mathrm{b} \rightarrow \mathrm{B}_{\mathrm{u}, \mathrm{d}}^{*} \rightarrow \mathrm{B}^{(*)} \pi\right)$ $=0.32 \pm 0.03$. The masses and widths of the $\mathrm{B}_{1}^{*}$ and $\mathrm{B}_{2}^{*}$ mesons are found to be

$M_{\mathrm{B}_{1}^{*}}=(5670 \pm 10$ (stat) $) \mathrm{MeV}$,

$\Gamma_{\mathrm{B}_{1}^{*}}=(70 \pm 21$ (stat) $) \mathrm{MeV}$,

$M_{\mathrm{B}_{2}^{*}}=(5768 \pm 5$ (stat) $) \mathrm{MeV}$,

$\Gamma_{\mathrm{B}_{2}^{*}}=(24 \pm 19$ (stat) $) \mathrm{MeV}$.

In addition, a total of $297 \pm 100$ events are in the high-mass Gaussian, denoted $\mathrm{B}^{\prime}$, corresponding to the branching fraction $\mathrm{Br}\left(\mathrm{b} \rightarrow \mathrm{B}^{\prime} \rightarrow \mathrm{B} \pi\right)=0.034 \pm$ 0.011 . The mass and Gaussian width are found to be $M_{\mathrm{B}^{\prime}}=(5936 \pm 22) \mathrm{MeV}$ and $\sigma_{\mathrm{B}^{\prime}}=(50 \pm 23) \mathrm{MeV}$. Table 3 presents the correlations of all free parameters in the fit. As this fit yields the best overall confidence level $(61.2 \%)$, compared to the other two fits described above, its values are chosen as the

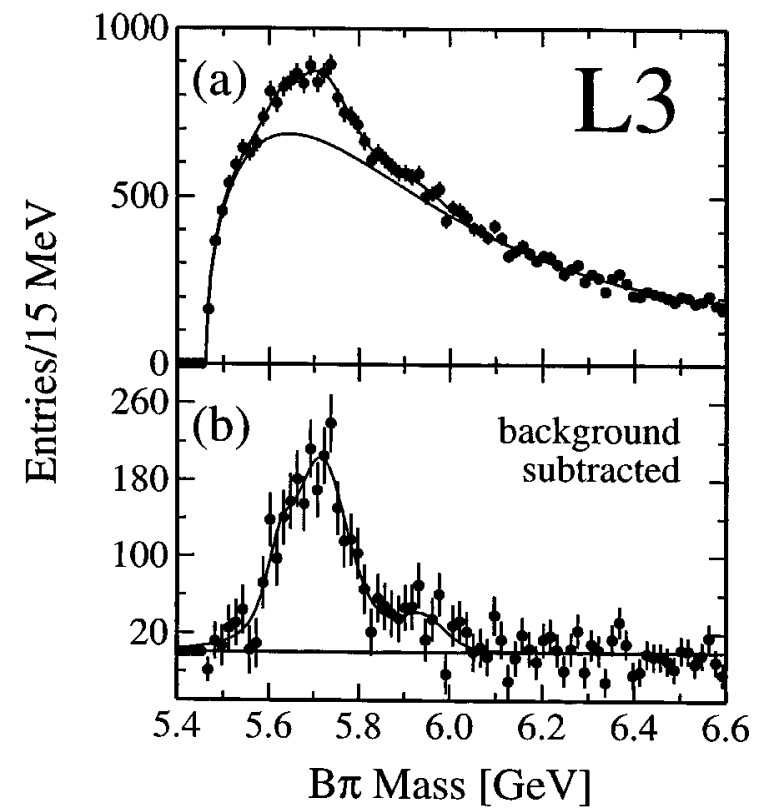

Fig. 3. (a) Fit to the data $B \pi$ mass distribution with the five-peak $\mathrm{B}^{* *}$ signal function, the Gaussian $\mathrm{B}^{\prime}$ signal function and the background function described in the text. (b) The resulting background-subtracted distribution.

results of the analysis. For comparison in the studies below, we refer to it as the "final fit."

The results of the final fit place the average mass of the $j_{\mathrm{q}}=3 / 2$ states $(98 \pm 11) \mathrm{MeV}$ higher than that of the $j_{\mathfrak{q}}=1 / 2$ states. This supports some theoretical predictions $[7,9]$ but is contrary to those which predict spin-orbit inversion $[11,12]$. To test the ability of the procedure to discriminate between the two possible cases, additional fits are performed. In these fits, the widths and relative production rates of the $j_{\mathrm{q}}=1 / 2$ and $j_{\mathrm{q}}=3 / 2$ states are varied, while the masses of the $j_{\mathrm{q}}=1 / 2$ states are constrained to be either equal to those of the $j_{q}=3 / 2$ states or up to $100 \mathrm{MeV}$ higher. In all cases, the fit confidence levels decrease as the mass of the $j_{\mathrm{q}}=1 / 2$ states is increased relative to that of the $j_{\mathrm{q}}=3 / 2$ states. The highest confidence level for a fit supporting spin-orbit inversion is found to be $8.3 \%$, relative to $61.2 \%$ for the final fit.

The fits performed above constrain both $M_{\mathrm{B}_{2}^{*}}-$ $M_{\mathrm{B}_{1}}$ and $M_{\mathrm{B}_{1}^{*}}-M_{\mathrm{B}_{0}^{*}}$ to $12 \mathrm{MeV}$. The results of varying this value to test the constraint as a source of systematic uncertainty are presented below. It was 
Table 3

Table of correlations of all free parameters in the final fit.

\begin{tabular}{|c|c|c|c|c|c|c|c|c|c|}
\hline Param. & $N_{\text {bgd }}$ & $N_{\mathrm{B}}{ }^{* *}$ & $M_{\mathrm{B}_{1}^{*}}$ & $\Gamma_{\mathrm{B}_{1}^{*}}$ & $M_{\mathrm{B}_{2}^{*}}$ & $\Gamma_{\mathrm{B}_{2}^{*}}$ & $N_{\mathrm{B}^{\prime}}$ & $M_{\mathrm{B}^{\prime}}$ & $\sigma_{\mathrm{B}^{\prime}}$ \\
\hline$N_{\mathrm{bgd}}$ & 1.000 & -0.579 & 0.144 & -0.140 & 0.100 & -0.231 & -0.606 & -0.157 & -0.432 \\
\hline$N_{\mathrm{B}}^{* *}$ & -0.579 & 1.000 & 0.004 & 0.468 & 0.133 & 0.607 & 0.050 & 0.289 & -0.022 \\
\hline$\Gamma_{\mathrm{B}_{1}^{*}}^{+}$ & -0.140 & 0.468 & 0.023 & 1.000 & -0.124 & 0.506 & -0.115 & 0.120 & -0.086 \\
\hline$M_{\mathrm{B}_{2}^{*}}$ & 0.100 & 0.133 & 0.276 & -0.124 & 1.000 & -0.041 & -0.355 & 0.247 & -0.362 \\
\hline$\Gamma_{\mathrm{B}_{2}^{*}}^{*}$ & -0.231 & 0.607 & 0.269 & 0.506 & -0.041 & 1.000 & -0.127 & 0.192 & -0.060 \\
\hline$\sigma_{\mathrm{B}^{\prime}}$ & -0.432 & -0.022 & -0.190 & -0.086 & -0.362 & -0.060 & 0.773 & 0.058 & 1.000 \\
\hline
\end{tabular}

suggested [25] to independently test the validity of the $j_{\mathrm{q}}=1 / 2$ mass-difference constraint since no corresponding measurements exist for the D-meson system, as they do for the $j_{\mathrm{q}}=3 / 2$ states. This test is performed by fitting the mass spectrum with $M_{\mathrm{B}_{1}^{*}}-$ $M_{\mathrm{B}_{0}^{*}}$ as an additional free parameter. The resulting fit, which has a $\chi^{2}$ of 63 for 66 degrees of freedom, yields masses, widths and branching fractions for the $\mathrm{B}_{1}^{*}, \mathrm{~B}_{2}^{*}$ and $\mathrm{B}^{\prime}$ which are consistent with the final fit. The mass difference of the $j_{\mathrm{q}}=1 / 2$ states is measured to be $M_{\mathrm{B}_{1}^{*}}-M_{\mathrm{B}_{0}^{*}}=(-39 \pm 71) \mathrm{MeV}$. While this value is consistent with the original constraint, the large error indicates that the method is insensitive to the mass difference of the $j_{\mathrm{q}}=1 / 2$ states and, conversely, that the results of the final fit are only weakly dependent on that constraint.

As an additional check, the mass spectrum is fit with the combination of a single Voigt function and the background function. The resulting fit has a $\chi^{2}$ of 114 for 76 degrees of freedom and describes the signal region poorly. A total of $1854 \pm 153$ events are in the signal, denoted $\mathrm{B}^{* *}$, corresponding to the branching fraction $\mathrm{Br}\left(\mathrm{b} \rightarrow \mathrm{B}^{* *} \rightarrow \mathrm{B}^{(*)} \pi\right)=0.21 \pm$ 0.02 . The mass and width are found to be $M_{\mathrm{B}^{* *}}=$ $(5713 \pm 2) \mathrm{MeV}$ and $\Gamma_{\mathrm{B}^{* *}}=(31 \pm 7) \mathrm{MeV}$. The hypothesis that the signal be the result of the decay of a single resonance is highly unlikely considering the low confidence level of the fit $(0.35 \%)$.

Sources of systematic uncertainty and their estimated contributions to the errors of the measured values are summarized in Table 4 . The b-hadron purity is varied from $92 \%$ to $96 \%$. This variation affects only the overall $\mathrm{B}^{* *}$ and $\mathrm{B}^{\prime}$ production branching fractions. The contribution to these errors due to uncertainty in $R_{\mathrm{b}}$, the branching ratio $\operatorname{Br}(\mathrm{Z} \rightarrow$ $\mathrm{b} \overline{\mathrm{b}}) / \operatorname{Br}(Z \rightarrow q \bar{q})$, is negligible.

Systematic effects due to background modelling are studied by varying the shape parameters of the background function (parameters $p_{2}$ through $p_{6}$ ) and by performing the fit with other background

Table 4

Sources of systematic uncertainty and their estimated contributions to the errors of the measured values. Effects due to correlations between the quantities are taken into consideration in the total error.

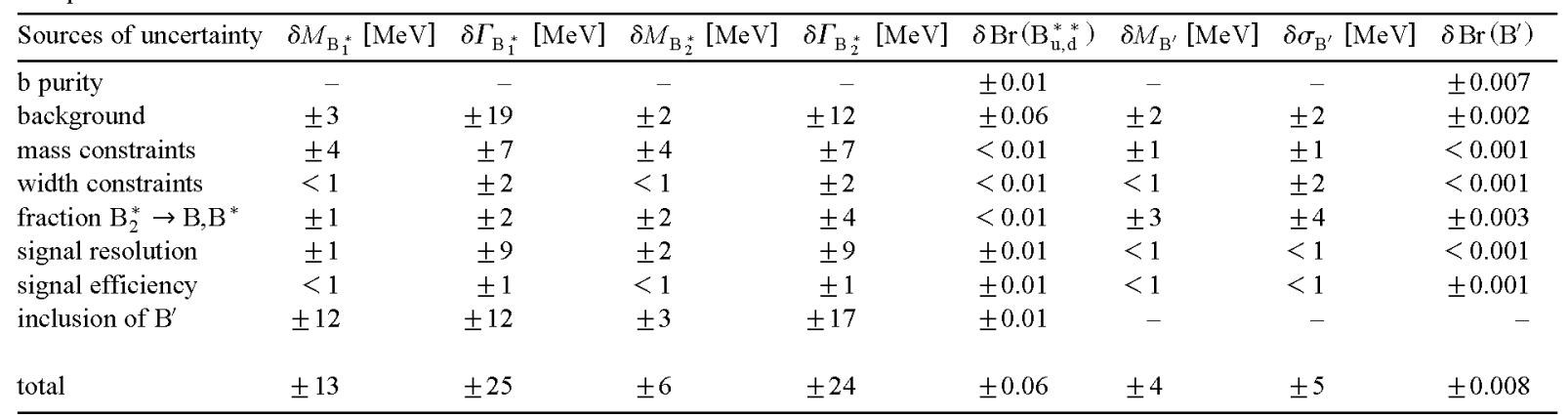


functions. Effects due to signal modelling are studied by varying the constraints on the masses, widths and relative production rates. The mass differences $M_{\mathrm{B}_{2}^{*}}$ $-M_{\mathrm{B}_{1}}$ and $M_{\mathrm{B}_{1}^{*}}-M_{\mathrm{B}_{0}^{*}}$ are each varied within the range 5-20 MeV, covering the full range of theoretical predictions. The ratios of the Breit-Wigner widths $\Gamma_{\mathrm{B}_{1}} / \Gamma_{\mathrm{B}_{2}^{*}}$ and $\Gamma_{\mathrm{B}_{0}^{*}} / \Gamma_{\mathrm{B}_{1}^{*}}$ are each varied between 0.8 and 1.0. This is to account for the possibility of a slight mass dependence due to the available phase space and the probability for $\mathrm{B}^{* *} \rightarrow \mathrm{B}^{(*)} \rho$ decays [26]. Finally, the fraction of $B_{2}^{*}$ mesons decaying to $\mathrm{B}^{*}$ mesons is varied between $1 / 3$ and $2 / 3$.

Effects due to uncertainty in the resolution function are estimated by varying the slope and offset of the linear parametrization. Effects due to mass-dependent variations in the signal efficiency are estimated by performing a linear parametrization of the Monte Carlo efficiencies, similar to that of the resolution, and then varying the corresponding slope and offset.

$\mathrm{B} \pi$ pairs from the decay $\mathrm{B}^{* *} \rightarrow \mathrm{B} \pi \pi$, for which only one of the pions is tagged, are studied as a possible source of resonant background. The resulting reflection is found to contribute insignificantly to the background in the low $Q$-value region. Similarly, $\mathrm{B}_{\mathrm{s}}^{* * *} \rightarrow \mathrm{BK}$ decays, for which the $\mathrm{K}$ is misidentified as a $\pi$, are found to contribute only slightly to the low $Q$-value region, and their effects are included in the background modelling uncertainty contribution.

\section{Conclusion}

Inclusively reconstructed $\mathrm{B}$ mesons in $\mathrm{Z} \rightarrow \mathrm{b} \overline{\mathrm{b}}$ events are combined with charged pions originating from the primary event vertex. A fit is performed to the $B \pi$ mass spectrum in the framework of Heavy Quark Symmetry [1] and under constraints common to several theoretical models. The results of the fit, which provide the first measurements of the masses and decay widths of the $\mathrm{B}_{1}^{*}\left(j_{\mathrm{q}}=1 / 2\right)$ and $\mathrm{B}_{2}^{*}$ $\left(j_{\mathrm{q}}=3 / 2\right)$ mesons, as well as the branching fraction for the combination of $l=1$ states, are

$$
\begin{aligned}
& M_{\mathrm{B}_{1}^{*}}=(5670 \pm 10 \text { (stat) } \pm 13 \text { (syst) }) \mathrm{MeV}, \\
& \Gamma_{\mathrm{B}_{1}^{*}}=(70 \pm 21 \text { (stat) } \pm 25 \text { (syst) }) \mathrm{MeV}, \\
& M_{\mathrm{B}_{2}^{*}}=(5768 \pm 5 \text { (stat) } \pm 6 \text { (syst) }) \mathrm{MeV},
\end{aligned}
$$

$$
\begin{aligned}
& \Gamma_{\mathrm{B}_{2}^{*}}=(24 \pm 19 \text { (stat) } \pm 24 \text { (syst) }) \mathrm{MeV}, \\
& \operatorname{Br}\left(\mathrm{b} \rightarrow \mathrm{B}_{\mathrm{u}, \mathrm{d}}^{* *} \rightarrow \mathrm{B}^{(*)} \pi\right) \\
& \quad=0.32 \pm 0.03 \text { (stat) } \pm 0.06 \text { (syst) } .
\end{aligned}
$$

In addition, an excess of events near $5.9-6.0 \mathrm{GeV}$ is interpreted as resulting from the decay $\mathrm{B}^{\prime} \rightarrow \mathrm{B}^{(*)} \pi$, where $\mathrm{B}^{\prime}$ denotes an excited B-meson state or mixture of states. From the same fit, the $\mathrm{B}^{\prime}$ mass, Gaussian width and branching fraction are

$$
\begin{aligned}
& M_{\mathrm{B}^{\prime}}=(5937 \pm 21 \text { (stat) } \pm 4 \text { (syst) }) \mathrm{MeV}, \\
& \sigma_{\mathrm{B}^{\prime}}=(50 \pm 22 \text { (stat) } \pm 5 \text { (syst) }) \mathrm{MeV}, \\
& \operatorname{Br}\left(\mathrm{b} \rightarrow \mathrm{B}^{\prime} \rightarrow \mathrm{B}^{(*)} \pi\right) \\
& \quad=0.034 \pm 0.011 \text { (stat) } \pm 0.008 \text { (syst). }
\end{aligned}
$$

For both branching fractions, isospin symmetry is employed to account for decays to neutral pions.

These results disfavor recent theoretical models proposing spin-orbit inversion [11,12], but agree well with earlier models [7,9] and provide support for Heavy Quark Effective Theory.

\section{Acknowledgements}

We wish to thank Chris Quigg and Adam Falk for sharing with us their invaluable expertise on the theoretical aspects of $\mathrm{B}^{* *}$ production and decays. In addition, we wish to express our gratitude to the CERN accelerator divisions for the excellent performance of the LEP machine. Finally, we acknowledge the contributions of the engineers and technicians who have participated in the construction and maintenance of this experiment.

\section{References}

[1] N. Isgur, M.B. Wise, Phys. Lett. B 232 (1989) 113; Phys. Lett. B 237 (1990) 527; S. Stone (Ed.), Their overview Heavy Quark Symmetry is included in B Decays, revised 2nd edition, World Scientific, 1994, p. 231; A.J. Buras, M. Lindner (Eds.), Heavy Flavors, World Scientific, 1992, p. 234.

[2] L3 Collaboration, M. Acciarri et al., Phys. Lett. B 345 (1995) 589

[3] DELPHI Collaboration, P. Abreu et al., Z. Phys. C 68 (1995) 353; ALEPH Collaboration, D. Buskulic et al., Z. Phys. C 69 
(1996) 393; OPAL Collaboration, K. Ackerstaff et al., Z. Phys. C 74 (1997) 413.

[4] J.L. Rosner, Comments Nucl. Part. Phys. 16 (1986) 109; N. Isgur, M.B. Wise, Phys. Rev. Lett. 66 (1991) 1130.

[5] M. Gronau, A. Nippe, J.L. Rosner, Phys. Rev. D 47 (1993) 1988.

[6] S. Godfrey, R. Kokoski, Phys. Rev. D 43 (1991) 1679.

[7] M. Gronau, J.L. Rosner, Phys. Rev. D 49 (1994) 254.

[8] E.J. Eichten, C.T. Hill, C. Quigg, Phys. Rev. Lett. 71 (1993) 4116; Fermilab-Conf-94/118-T, 1994.

[9] S.N. Gupta, J.M. Johnson, Phys. Rev. D 51 (1995) 168.

[10] A.F. Falk, T. Mehen, Phys. Rev. D 53 (1996) 231.

[11] N. Isgur, Phys. Rev. D 57 (1998) 4041.

[12] D. Ebert, V.O. Galkin, R.N. Faustov, Phys. Rev. D 57 (1998) 5663.

[13] OPAL Collaboration, R. Akers et al., Z. Phys. C 66 (1995) 19; DELPHI Collaboration, P. Abreu et al., Phys. Lett. B 345 (1995) 598; ALEPH Collaboration, D. Buskulic et al., Z. Phys. C 69 (1996) 393.

[14] ALEPH Collaboration, R. Barate et al, Phys. Lett. B 425 (1998) 215.

[15] L3 Collaboration, B. Adeva et al., Nucl. Instr. and Meth. A 289 (1990) 35; O. Adriani et al., Nucl. Instr. and Meth. A 302 (1991) 53; B. Adeva et al., Nucl. Instr. and Meth. A 323 (1992) 109; K. Deiters et al., Nucl. Instr. and Meth. A 323 (1992) 162; M. Chemarin et al., Nucl. Instr. and Meth. A 349 (1994) 345; M. Acciarri et al., Nucl. Instr. and Meth. A 351 (1994) 300; G. Basti et al., Nucl. Instr. and Meth. A 374 (1996) 293; A. Adam et al., Nucl. Instr. and Meth. A 383 (1996) 342 .

[16] L3 Collaboration, O. Adriani et al., Phys. Lett. B 307 (1993) 237.

[17] J. Branson et al., L3 Note 2108, June 24, 1997; L3 Collaboration, M. Acciarri et al., Phys. Lett. B 411 (1997) 373.

[18] T. Sjöstrand, Comp. Phys. Comm. 39 (1986) 347; T. Sjöstrand, M. Bengtsson, Comput. Phys. Commun. 43 (1987) 367; T. Sjöstrand, Comp. Phys. Comm. 82 (1994) 74; T. Sjöstrand, Lund and CERN Preprints LU TP 95-20/CERNTH.7112/93 (revised, 1995). The parameters of JETSET have been tuned in order to reproduce event shape variables as seen by the L3 detector. The production of particles (tensor mesons) with orbital angular momentum is enabled. For the tuning see L3 Collaboration, B. Adeva et al., Z. Phys. C 55 (1992) 39.

[19] The L3 detector simulation is based on GEANT Version 3.15; see R. Brun et al., GEANT 3, CERN DD/EE/84-1 (revised 1987) and the GHEISHA program (H. Fesefeld, RWTH Aachen Report PITHA 85/02 (1985)) for the simulation of hadronic interactions.

[20] CDF Collaboration, F. Abe et al., Phys. Rev. Lett. 80 (1998) 2057.

[21] J. Swain, L. Taylor, Nucl. Instr. and Meth. 411 (1998) 153.

[22] A description of the bifurcated Gaussian function used in the fit can be found in: Ian C. Brock, Mn_Fit: A Fitting and Plotting Package Using MINUIT, L3 Note 918, PPE Div., CERN, 1993

[23] ARGUS Collaboration, H. Albrecht et al., Phys. Rev. Lett. 56 (1986) 549; H. Albrecht et al., Phys. Lett. B 221 (1989) 422; B 231 (1989) 208; Z. Phys. C 69 (1996) 405; CLEO Collaboration, T. Bowcock et al., Phys. Rev. D 41 (1990) 774; J. Alexander et al., Phys. Lett. B 303 (1993) 377; P. Avery et al., Phys. Lett. B 331 (1994) 236; T. Bergfeld et al., Phys. Lett. B 340 (1994) 194; Y. Kubota et al., Phys. Rev. Lett. 72 (1994) 1972; E691 Collaboration, P.E. Karchin et al., Phys. Rev. Lett. 62 (1989) 1717; E687 Collaboration, P.L. Frabetti et al., Phys. Rev. Lett. 72 (1994) 324; BEBC Collaboration, W. Burkot et al., Z. Phys. C 61 (1994) 563; OPAL Collaboration, R. Akers et al., Z. Phys. C 67 (1995) 57; K. Ackerstaff et al., Z. Phys. C 76 (1997) 425; ALEPH Collaboration, D. Buskulic et al., Phys. Lett. B 345 (1994) 103; D. Buskulic et al., Z. Phys. C 62 (1994) 1; C 73 (1997) 601.

[24] M. Feindt, O. Podobrin, DELPHI Collaboration, Submitted to the ICHEP Conference, Warsaw, July 1996, ICHEP96 Ref. pa01-021.

[25] Private communication with A.F. Falk, March, 1999.

[26] Private communication with C. Quigg, July, 1998. In addition to effects of phase space in the HQET predictions, an effective increase of the decay width may occur if the $Q$ value is large enough to include the tail of the decay $\mathrm{B}^{* *} \rightarrow$ $\mathrm{B} \rho$. Both of these effects would cause higher-mass states to have larger decay widths. 